\title{
Intra-layer Synchronization in a Duplex Networks with Noise
}

\author{
Jinsen Zhuang ${ }^{1}$, Yan Zhou ${ }^{1}$, and Yonghui Xia ${ }^{2}$ \\ ${ }^{1}$ Huaqiao University \\ ${ }^{2}$ zhejiang Normal University
}

June 9,2021

\begin{abstract}
This paper concerns the impact of stochastic perturbations on the intra-layer synchronization of the duplex networks. A duplex network contains two layers $([1,2])$. Different from the previous works, environmental noise is introduced into the dynamical system of the duplex network. We incorporate both the inter-layer delay and the intra-layer delay into the dynamical system. Both of the delays are time-varying. However, the paper [1] only considered the intra-layer delays and they are assumed as the constants. While the paper [2] did not consider the inter-layer delay or intra-layer delay. When the system does not achieve automatic intra-layer synchronization, we introduce two controllers: one is the state-feedback controller, the other is the adaptive state-feedback controller. Interestingly, we find that the intra-layer synchronization will achieve automatically if the inter-layer coupling strength $\$ c_{-} 1 \$$ is large enough when the time-varying inter-layer delays are absent. Finally, some interesting simulation results are obtained for the Chua-Chua chaotic system with application of our theoretic results, which show the feasibility effectiveness of our control schemes.
\end{abstract}

\section{Hosted file}

DuplexN-2021-5-27-MMA.pdf available at https://authorea.com/users/418875/articles/525483intra-layer-synchronization-in-a-duplex-networks-with-noise 